$(-1,0,0),\left(-\alpha_{1}, \pm\left(1-\alpha_{1}^{2}\right)^{\frac{1}{2}}, 0\right),\left(\alpha_{2}, \pm\left(1-\alpha_{2}^{2}\right)^{\frac{1}{2}}, 0\right),\left(-\alpha_{3}, \pm \alpha_{4}, \pm\left(1-\alpha_{3}{ }^{2}-\alpha_{4}^{2}\right)^{\frac{1}{2}}\right)$, $\left(\left(1-\alpha_{5}^{2}\right)^{\frac{1}{2}}, 0, \pm \alpha_{5}\right)$, with five degrees of freedom $\alpha_{i}$.

Thus we observe the final configuration for $n=9(n=11)$ and regenerate taking initially $\alpha=.699929\left(\alpha_{1}=.505694, \alpha_{2}=.507286, \alpha_{3}=.089001, \alpha_{4}=492006\right.$, $\left.\alpha_{5}=.571672\right)$. These are crude guesses based on averages of diagonal lengths. In fact $V$ now increases to 25.76028061 (40.64744085) which brings us back to the twenty-third (eleventh) step of the descent process! We are further ahead, however, by virtue of the new self-preserving symmetry. In fact in five (thirty) additional circuits of the main loop we find $V$ has come to the even lower value $25.75998651(40.59645048)$ probably correct to eight decimal places at which point the particle positions change by $\xi<10^{-5}=\epsilon$. The final values of the coordinates of the configuration are given by $\alpha=.703648\left(\alpha_{1}=.515358, \alpha_{2}=.552626\right.$, $\left.\alpha_{3}=.168322, \alpha_{4}=.488923, \alpha_{5}=.591930\right)$, probably correct to five decimal places, in a total of about 15(60) minutes of computing time.

The author is indebted to G. Pólya and J. L. Ullman for illuminating discussions and to Don Hart and George Ryckman of the General Motors Technical Center at Warren, Michigan for kindly making the IBM 701 available.

Department of Mathematics

HaRvey Cohn

Washington University

St. Louis, Missouri

1. L. FöPPL, "Stabile Anordnungen von Elektronen im Atom," J. für die Reine Angew. Math., v. 141, 1912, p. 251-301.

\title{
An Iterative Method for Taylor Expansion of Rational Functions, and Applications
}

1. The iteration and some applications. A simple iterative procedure can be set up for determining the coefficients of the Taylor expansion of a rational function at any point in the complex plane other than one of the singularities of the function. This procedure would have many applications, particularly in the evaluation of inverse Laplace transforms. We shall discuss the general method first, and then some applications, followed by a discussion of truncation and round-off errors.

Suppose $f(s)$ is a rational function, that is,

$$
f(s)=\frac{p(s)}{q(s)}
$$

where $p(s)$ and $q(s)$ are polynomials. We shall assume that we have $p(s)$ and $q(s)$ expressed in powers of $s$, rather than in their factored form.

Now consider any point " $a$ " in the complex plane, with the restriction that $q(a) \neq 0$. Then by Taylor's theorem, within the circle of convergence around " $a$ ", we have

$$
f(s)=\frac{p(s)}{q(s)}=\alpha_{0}+\alpha_{1}(s-a)+\alpha_{2}(s-a)^{2}+\cdots
$$


Multiplying (2) by $q(s)$,

$$
p(s)=q(s)\left[\alpha_{0}+\alpha_{1}(s-a)+\alpha_{2}(s-a)^{2}+\cdots\right] .
$$

Setting $s=a$, we see that

$$
\alpha_{0}=\frac{p(a)}{q(a)} .
$$

Having thus determined $\alpha_{0}$, we define the polynomial

$$
\phi_{1}(s)=p(s)-\alpha_{0} q(s) .
$$

Clearly $\phi_{1}(a)=0$, so " $a$ " is a root of $\phi_{1}(s)$. Thus we can define another polynomial,

$$
p_{1}(s)=\frac{\phi_{1}(s)}{s-a} .
$$

Then from (3) and (5), we have

$$
p_{1}(s)=q(s)\left[\alpha_{1}+\alpha_{2}(s-a)+\alpha_{3}(s-a)^{2}+\cdots\right]
$$

Comparing equation (7) with (3), we see that we have replaced $p(s)$ by $p_{1}(s)$, and $\alpha_{j}$ by $\alpha_{j+1}(j=0,1,2,3, \cdots)$. Continuing as before we get

$$
\alpha_{1}=\frac{p_{1}(a)}{q(a)},
$$

$$
\begin{aligned}
& \phi_{2}(s)=p_{1}(s)-\alpha_{1} q(s) \\
& p_{2}(s)=\frac{\phi_{2}(s)}{s-a} \\
& \alpha_{2}=\frac{p_{2}(a)}{q(a)}
\end{aligned}
$$

and so on

More precisely, defining $p_{0}(s)=p(s)$, we get the recursive relationships

$$
\begin{aligned}
\alpha_{j} & =\frac{p_{j}(a)}{q(a)}, \\
\phi_{j+1}(s) & =p_{j}(s)-\alpha_{j} q(s),
\end{aligned}
$$

and

$$
p_{j+1}(s)=\frac{\phi_{j+1}(s)}{s-a}
$$

where $j=0,1,2,3$, etc. 
This method is particularly suitable for digital computation for several reasons. One is that since the process is recursive, it is just as easy to program for a large number of the $\alpha_{j}$ as for only a few. Also let us examine the equations (12), (13) and (14) in more detail. We start with the coefficients of the polynomials $p_{0}(s)$ (i.e., $p(s)$ ), and $q(s)$. We can obtain $p_{0}(a)$ by performing the synthetic division of $p_{0}(s)$ by $(s-a)$ and taking the remainder. We obtain $q(a)$ by the same method, and then perform the straight division to get $\alpha_{0}$. The coefficients of $\phi_{1}(s)$ are easily calculated, since they are obtained by subtracting $\alpha_{0}$ times the coefficients of $q(s)$ from the corresponding coefficients of $p_{0}(s)$. We now again use our synthetic division process, dividing $\phi_{1}(s)$ by $(s-a)$, taking the quotient for $p_{1}(s)$ and inserting an automatic check to make sure that the remainder is zero (to within round-off errors, etc.). Then we go back to equation (12), and compute $\alpha_{1}=p_{1}(a) / q(a)$. We evaluate $p_{1}(a)$ by the synthetic division method again and of course $q(a)$ is now already calculated from the first round, and does not have to be calculated again. Thus the calculation continues, and a counter can be used to control the number of iterations, depending on the number of coefficients $\alpha_{j}$ that is desired.

Thus we have reduced the problem primarily to synthetic division. Of course in general we will have to use sub-routines for complex arithmetic, although if the original polynomials have real coefficients and we are only interested in expanding about points on the real axis, this will not be necessary.

We now consider some applications of the above method. First suppose we wish to find the inverse Laplace transform of a rational function

$$
\bar{F}(s)=\frac{N(s)}{D(s)},
$$

where $N(s)$ and $D(s)$ are polynomials, the degree of $D(s)$ being higher than that of $N(s)$. Suppose also that we know the roots of $D(s)$, so that

$$
D(s)=K\left(s-s_{1}\right)^{n_{1}}\left(s-s_{2}\right)^{n_{2}} \cdots\left(s-s_{m}\right)^{n_{m}} .
$$

Then we can expand $\bar{F}(s)$ in partial fractions:

$$
\begin{aligned}
\bar{F}(s)=\frac{A_{11}}{\left(s-s_{1}\right)}+\frac{A_{12}}{\left(s-s_{1}\right)^{2}}+\cdots+ & \frac{A_{1 n_{1}}}{\left(s-s_{1}\right)^{n_{1}}}+\frac{A_{21}}{\left(s-s_{2}\right)} \\
& +\frac{A_{22}}{\left(s-s_{2}\right)^{2}}+\cdots+\frac{A_{m n_{m}}}{\left(s-s_{m}\right)^{n_{m}}},
\end{aligned}
$$

or in contracted form

$$
\bar{F}(s)=\sum_{i=1}^{m} \sum_{j=1}^{n_{i}} \frac{A_{i j}}{\left(s-s_{i}\right)^{j}} .
$$

Once we have $\bar{F}(s)$ expanded in this fashion we can easily find the inverse transform, since the inverse transform of $\frac{1}{(s-b)^{k}}$ is $\frac{t^{k-1} e^{b t}}{(k-1) !}(k=1,2,3 \cdots)$, $(0 !=1)$. The problem is to find the coefficients $A_{i j}$. Clearly if we can find the coefficients corresponding to $s_{1}$, i.e., $A_{1 j}$, we can find the rest in the same manner. 
Thus let us break up the expression of equation (17) into the contribution from the root $s_{1}$, and the remainder. We get

$$
\bar{F}(s)=\frac{A_{11}}{s-s_{1}}+\frac{A_{12}}{\left(s-s_{1}\right)^{2}}+\cdots+\frac{A_{1 n_{1}}}{\left(s-s_{1}\right)^{n_{1}}}+h(s),
$$

and $h(s)$ is analytic at the point $s_{1}$. Returning to equation (15) now, we write

$$
\bar{F}(s)=\frac{N(s)}{\left(s-s_{1}\right)^{n_{1}} D_{1}(s)},
$$

where the polynomial

$$
D_{1}(s)=\frac{D(s)}{\left(s-s_{1}\right)^{n_{1}}}
$$

Clearly $D_{1}\left(s_{1}\right) \neq 0$. Then multiplying (18) by $\left(s-s_{1}\right)^{n_{1}}$, and using (19), we get

$$
\begin{aligned}
& \frac{N(s)}{D_{1}(s)}=A_{1 n_{1}}+A_{1\left(n_{1}-1\right)}\left(s-s_{1}\right)+A_{1\left(n_{1}-2\right)}\left(s-s_{1}\right)^{2}+\cdots \\
& \quad+A_{12}\left(s-s_{1}\right)^{n_{1}-2}+A_{11}\left(s-s_{1}\right)^{n_{1}-1}+\left(s-s_{1}\right)^{n_{1}} h(s) .
\end{aligned}
$$

Thus $A_{1 n_{1}}, A_{1\left(n_{1}-1\right)}, \cdots A_{12}, A_{11}$, are the first $n_{1}$ Taylor coefficients for the expansion of $\frac{N(s)}{D_{1}(s)}$ around the point $s_{1}$, and we can find them by the method we have described. We would wish to have $D_{1}(s)$ in powers of $s$ rather than in the factored form, so we would start with $D(s)$ expressed in powers of $s$. Then we perform the synthetic division by $\left(s-s_{1}\right) n_{1}$ times to get the coefficients of $D_{1}(s)$. The remainder should be zero each time, and as before this fact can be used for error checking.

In most cases, $\bar{F}(s)$ will have only simple poles; i.e., we would have $n_{1}=n_{2} \cdots$ $=n_{m}=1$. In that case the method described by Titus [1] is essentially the same as this one, perhaps even a little simpler. However, Titus' method gets considerably more cumbersome when treating multiple poles, and since the method we have described here works equally well for multiple poles, it would seem preferable in this respect, provided the computing machine used has sufficient internal memory for the incorporation of the necessary subroutines. Furthermore the routine for the Taylor expansion of rational functions could be used for other applications such as will be described below, as well as for partial fraction expansion.

In the next example we again consider

$$
\bar{F}(s)=\frac{N(s)}{D(s)}
$$

where the degree of $N(s)$ is lower than that of $D(s)$. However, suppose we wish to have the inverse Laplace transform, $F(t)$, as a power series in $t$. To do this we expand $\bar{F}(s)$ around the point at infinity, using a method due to Heaviside [2]. 
First we substitute $s=1 / z$, and then multiply numerator and denominator by $z^{N}$, where $N$ is the degree of $D(s)$, obtaining

$$
\bar{F}\left(\frac{1}{z}\right)=f(z)=\frac{p(z)}{q(z)}
$$

where $p(z)$ and $q(z)$ are polynomials. Also (due to the fact that $N(s)$ is of lower degree than $D(s)$ )

$$
f(0)=0,
$$

so that we can factor out $z$ and write

$$
f(z)=z g(z)
$$

Then if

$$
\begin{aligned}
& g(z)=\sum_{j=0}^{\infty} \alpha_{j} z^{j} \\
& F(t)=\sum_{j=0}^{\infty} \frac{\alpha_{j} t^{j}}{j !} \quad(t>0) .
\end{aligned}
$$

Thus we can use the method we have described to obtain the coefficients $\alpha_{j}$ in (25), and substitution in (26) will give the series for $F(t)$. The rate of convergence of this series will be discussed later in some detail under the subject of truncation errors.

The formulae for $\alpha_{j}$ in this case can be simplified somewhat. Suppose the function $\bar{F}(s)$ is in the form

$$
\bar{F}(s)=\frac{a_{0} s^{N-1}+a_{1} s^{N-2}+\cdots+a_{N-2} s+a_{N-1}}{s^{N}+b_{1} s^{N-1}+b_{2} s^{N-2}+\cdots+b_{N-1} s+b_{N}} .
$$

(It can always be expressed in the above form, with the coefficient of the highest degree term, $s^{N}$, equal to unity.) Then from (22) and (24) we obtain

$$
g(z)=\frac{P_{0}(z)}{Q(z)}
$$

where

$$
P_{0}(z)=a_{0}+a_{1} z+\cdots+a_{N-1} z^{N-1}
$$

and

$$
Q(z)=1+b_{1} z+b_{2} z^{2}+\cdots+b_{N} z^{N}
$$

It is not difficult to show from (12), (13), and (14) that the coefficients $\alpha_{j}$ in (25) are given by

$$
\begin{aligned}
\alpha_{j} & =a_{0, j} \\
a_{k, j+1} & =a_{k+1, j}-\alpha_{j} b_{k+1}, \quad 0 \leq k \leq N-2, \\
a_{N-1, j+1} & =-\alpha_{j} b_{N}
\end{aligned}
$$


where

$$
a_{m, 0}=a_{m}, \quad 0 \leq m \leq N-1 .
$$

One advantage of the above method is that it is not necessary to know the roots of the denominator of $\bar{F}(s)$.

The third application we shall mention is one from probability theory. Suppose we have a random variable $X$, which can take on the values $0,1,2,3$, etc. (and no other values) and that it takes on these values with probability $P_{0}, P_{1}, P_{2}, P_{3}$, etc. Thus $P_{j}(j=0,1,2, \cdots)$ gives the "probability distribution" of the random variable $X$.

In dealing with such distributions, especially when treating more than one of them at a time, it often is convenient to treat not the probability distribution itself, but its "generating function," $P(s)$. The generating function is precisely that function which, when expanded in its Taylor series around $s=0$, has Taylor coefficients $P_{0}, P_{1}, P_{2}$, etc. Furthermore, these generating functions are of ten, though not always, rational functions. In such cases we can see that the method we have described would be very useful in obtaining a probability distribution from its generating function.

A good discussion of generating functions is given in [3], chapter 11.

There are also many other possible applications for the procedure that has been presented here. For example it might simply be used to calculate one of the derivatives of a rational function, since the $n$-th derivative at a point " $a$ " is given by

$$
\left[\frac{d^{n} f(s)}{d s^{n}}\right]_{\delta=a}=n ! \alpha_{n}
$$

where $\alpha_{n}$ is as before coefficient of $(s-a)^{n}$ in the Taylor expansion about " $a$ ".

2. Truncation and round-off errors. We come next to the important question of errors in the above method. We shall consider first truncation errors, due to terminating the series (2) after a finite number of terms. To do this we assume that each term in the series is evaluated exactly, without round-off error, leaving the discussion of round-off errors till later.

First we note an important theorem. Let $\rho$ be the radius of convergence of the series (2) about " $a$." Let $0<r_{0}<\rho, 0<\theta<1$, and let $r_{1}=\theta r_{0}$. Let $M$ be an upper bound of $|f(s)|$ on the circle, $|s-a|=r_{0}$. Then if the remainder

$$
\begin{aligned}
& R_{n}(s)=\sum_{j=n}^{\infty} \alpha_{j}(s-a)^{j}, \\
&\left|R_{n}(s)\right| \leq \frac{M \theta^{n}}{1-\theta}
\end{aligned}
$$

whenever $|s-a| \leq r_{1}$. For a derivation of this condition see [4], chapter VI.

This gives uniform convergence on the region, $|s-a| \leq r_{1}$, and we can make $\left|R_{n}(s)\right|$ less than any given positive number $\mu$, over the region, by choosing 
$n$ such that

$$
n>\frac{\ln \left[\frac{M}{\mu(1-\theta)}\right]}{\ln \left(\frac{1}{\theta}\right)}
$$

where "In" denotes the natural logarithm.

In the first example we have discussed, pertaining to partial fraction expansion, the question of truncation errors does not arise, since in this cas we are interested only in a finite number of the terms. In the second example however, the power series expansion for $F(t)$, the truncation errors are important. Here we cannot use (38) directly because we are interested in the truncation errors in equation (26), rather than (25). In order to study this case we first define

$$
h(z)=\frac{g(z)-g(0)}{z} .
$$

Then

$$
h(z)=\frac{P_{1}(z)}{Q(z)},
$$

where $Q(z)$ is given by equation (30) and

$$
P_{1}(z)=c_{0}+c_{1} z+\cdots+c_{N-1} z^{N-1}
$$

where

$$
\begin{aligned}
& c_{0}=a_{1}-a_{0} b_{1} \\
& c_{1}=a_{2}-a_{0} b_{2} \\
& \cdot \\
& \cdot \\
& \cdot \\
& c_{N-2}=a_{N-1}-a_{0} b_{N-1} \\
& c_{N-1}=-a_{0} b_{N} .
\end{aligned}
$$

(The coefficients $c_{k}$ above are the same as the quantities $a_{k, 1}$ in equations (32) and (33).) The MacLaurin series for $h(z)$ is given by

$$
h(z)=\sum_{j=0}^{\infty} \beta_{j} z^{\prime}
$$

and from (25) and (39) we see that

$$
\beta_{j}=\alpha_{j+1} \quad(j=0,1,2, \cdots)
$$

Next we let $r_{1}$ be a positive number, as yet unspecified except that it is less than the modulus of each of the zeros of $Q(z)$. Then (43) is uniformly convergent for $|z| \leq r_{1}$.

Now suppose we wish to take enough terms of (26) to be accurate within $\pm \epsilon$ on the interval $0<t \leq t_{0}$. We can find $n_{0}$ such that when $n>n_{0}$

$$
\left|R_{n}(z)\right|<\frac{2 \epsilon}{r_{1}} e^{-\frac{t_{0}}{r_{1}}}
$$


for $|z| \leq r_{1}$, where

$$
R_{n}(z)=\sum_{j=n}^{\infty} \beta_{j} z^{j}
$$

Then, if

$$
F_{n}(t)=\sum_{j=0}^{n} \frac{\alpha_{j} t^{j}}{j !}
$$

we have, by the inversion theorem, using (22), (24), (25), and (44), $(t>0)$,

$$
\begin{aligned}
& \left|F(t)-F_{n}(t)\right|=\frac{1}{2 \pi}\left|\int_{\frac{1}{r_{1}}-i \infty}^{\frac{1}{r_{1}}+i \infty} e^{s t} \bar{F}(s) d s-\sum_{j=0}^{\infty} \alpha_{j} \int_{\frac{1}{r_{1}}-i \infty}^{\frac{1}{r_{1}}+i \infty} \frac{e^{s t}}{s^{j+1}} d s\right| \\
& =\frac{1}{2 \pi}\left|\int_{\frac{1}{r_{1}}-i \infty}^{\frac{1}{r_{1}}+i \infty}\left[\sum_{j=0}^{\infty} \frac{\alpha_{j}}{s^{j+1}}-\sum_{j=0}^{n} \frac{\alpha_{j}}{s^{j+1}}\right] e^{s t} d s\right|=\frac{1}{2 \pi}\left|\int_{\frac{1}{r_{1}}-i \infty}^{\frac{1}{r_{1}}+i \infty} \frac{e^{s t}}{s^{2}} \sum_{j=n}^{\infty} \frac{\beta_{j}}{s^{j}} d s\right| \\
& =\frac{1}{2 \pi}\left|\int_{\frac{1}{r_{1}}-i \infty}^{\frac{1}{r_{1}}+i \infty} \frac{e^{s t} R_{n}\left(\frac{1}{s}\right)}{s^{2}} d s\right|=\frac{1}{2 \pi} \mid \int_{-\infty}^{\infty} \frac{e^{\frac{t}{r_{1}}+i y t}}{R_{n}\left[\left(\frac{1}{r_{1}}+i y\right)^{-1}\right] i d y \mid}
\end{aligned}
$$

Thus if $0<t \leq t_{0}$, and $n>n_{0}$, we obtain

$$
\left|F(t)-F_{n}(t)\right|<\frac{1}{2 \pi} \frac{2 \epsilon}{r_{1}} e^{\frac{t-t_{0}}{r_{1}}} \int_{-\infty}^{\infty} \frac{d y}{y^{2}+\frac{1}{r_{1}^{2}}} \leq \epsilon .
$$

The above approach is equivalent to that used in [2], chapter XIII to prove the validity of equation (26), in the more general case where $\bar{F}(s)$ is analytic at infinity, but is not necessarily a rational function.

We now wish to make a specific choice of $r_{1}$ and an appropriate $n_{0}$. In order to use the theorem stated above for truncation errors, we first pick $r_{0}$ and then make $r_{1}<r_{0}$ (both being less then the radius of convergence of (43)). First let $B$ be the maximum of the quantities $\left|b_{1}\right|,\left|b_{2}\right|, \cdots\left|b_{N}\right|$, obtained from equation (30). Then we will choose

$$
r_{0}=\frac{1}{1+B}
$$

For $|z| \leq r_{0}$

$$
\begin{aligned}
|Q(z)| \geq 1-B\left[\frac{1}{1+B}+\right. & \left.\left(\frac{1}{1+B}\right)^{2}+\cdots\left(\frac{1}{1+B}\right)^{N}\right] \\
& =1-\frac{B}{1+B} \frac{1-\left(\frac{1}{1+B}\right)^{N}}{1-\left(\frac{1}{1+B}\right)}=\frac{1}{(1+B)^{N}},
\end{aligned}
$$

so that our choice of $r_{0}$ is a satisfactory one. 
Next we must find $M$, an upper bound, of $|h(z)|$ on the circle $|z|=r_{0}$. From (40), (41), and (42), we see that each of the coefficients in the numerator, $P_{1}(z)$, is less than $A(1+B)$, where $A$ is the maximum of the quantities $\left|a_{1}\right|$, $\left|a_{2}\right|, \cdots\left|a_{N-1}\right|$, so that for $|z|=r_{0}=\frac{1}{1+B}$,

$$
\begin{array}{r}
\left|P_{1}(z)\right| \leq A(1+B)\left[1+\frac{1}{1+B}+\left(\frac{1}{1+B}\right)^{2}+\cdots+\left(\frac{1}{1+B}\right)^{N-1}\right] \\
=A(1+B) \frac{1-\left(\frac{1}{1+B}\right)^{N}}{1-\frac{1}{1+B}} .
\end{array}
$$

Then using (51), we have for $|z|=r_{0}$

$$
|h(z)| \leq A(1+B)^{N+1} \frac{1-\left(\frac{1}{1+B}\right)^{N}}{1-\frac{1}{1+B}}=\frac{A(1+B)^{2}\left[(1+B)^{N}-1\right]}{B}
$$

so we choose the right hand side of (53) for $M$. Letting

$$
r_{1}=\theta r_{0}=\frac{\theta}{1+B}, \quad 0<\theta<1
$$

(45) becomes

$$
\left|R_{n}(z)\right|<\frac{2 \epsilon(1+B)}{\theta} e^{-\frac{(1+B) t_{0}}{\theta}}
$$

Thus, from (38), (55) will be satisfied for $|z| \leq r_{1}$ if $n>n_{0}$, where

$$
\begin{aligned}
n_{0} & =\frac{\ln \left[\frac{A(1+B)^{2}\left[(1+B)^{N}-1\right]}{B(1-\theta)} \cdot \frac{\theta e^{\frac{(1+B) t_{0}}{\theta}}}{2 \epsilon(1+B)}\right]}{\ln \left(\frac{1}{\theta}\right)} \\
= & \frac{(1+B) t_{0}}{\theta \ln \left(\frac{1}{\theta}\right)}+\frac{\ln \left[\frac{A(1+B)\left[(1+B)^{N}-1\right] \theta}{2 B(1-\theta) \epsilon}\right]}{\ln \left(\frac{1}{\theta}\right)}
\end{aligned}
$$

and $A$ and $B$ are the maximum absolute values of the quantities $a_{j}$ and $b_{j}$ respectively in equation (27) ( $N$ being the degree of the denominator of $\bar{F}(s)$ ).

Thus if $n>n_{0}$, defined by (56), we will have

$$
\left|F(t)-F_{n}(t)\right|<\epsilon, \quad\left(0<t \leq t_{0}\right)
$$


$\theta$ can be any number between 0 and 1 , but we might choose it so as to make the term in $t_{0}$ a minimum. If we do this we obtain $\theta=e^{-1}$, so that (56) becomes

$$
n_{0}=e(1+B) t_{0}+\ln \left[\frac{A(1+B)\left[(1+B)^{N}-1\right]}{2 B(e-1) \epsilon}\right] \text {. }
$$

Finally we consider the third example of the probability generating function

$$
P(s)=\sum_{j=0}^{\infty} P_{j} s^{j}
$$

Since we must have $\sum_{j=0}^{\infty} P_{j}=1$, the radius of convergence, $\rho$, is either greater than or equal to unity. The probability that $X \geq n$ is

$$
R_{n}(1)=\sum_{j=n}^{\infty} P_{j}
$$

One might often be interested in choosing $n$ large enough so that this quantity is less than some preassigned small number. If $\rho>1$ this can be done by the general theory we have described. If $\rho=1$ however we see that this theory does not apply and the problem becomes somewhat more difficult.

We now come to the general problem of numerical round-off errors. If one is summing a certain number of terms of a series and one can estimate the accuracy of each term, it is not difficult to study the error in the sum. The more serious problem, in our case, appears to be the possibility of cumulative errors in the calculation of the $\alpha_{j}$ themselves.

Let us assume that we are using a floating decimal computing system which retains four significant figures in the mantissa. Consider the calculation

$$
x=[4.790-4.751] \div 1.783
$$

where we perform the subtraction first, and then the division. The computer will give

$$
x=2.187 \times 10^{-2} \text {. }
$$

Next consider the equivalent calculation

$$
x^{\prime}=(2.507 \times 4.790-2.507 \times 4.751) \div(2.507 \times 1.783)
$$

3 where we evaluate each term in the numerator, subtract, evaluate the denominator, and finally divide. The computer will give

$$
x^{\prime}=2.237 \times 10^{-2} \text {. }
$$

In this case we could see beforehand that the answer would only be valid to two significant figures, and we note that $x$ and $x^{\prime}$ agree to two figures but no further.

This illustrates the point that round-off errors can usually be detected by a duplicate calculation by a modified routine, although it is difficult to obtain hard 
and fast rules for doing so. In the above example, for instance, if we substitute some other number, $k$ say, for 2.507 , the desired effect does not occur for all values of $k$. An integral power of ten will not work (since we are assuming rounding-off is done in the decimal system). Also one finds experimentally in this case that $k=1.001$ and $k=2$ are unsatisfactory choices.

In our present study we might use a transformation of the type

$$
s=k s^{\prime}
$$

and re-evaluate the $\alpha_{j}$ in equation (2) by expanding the function

$$
\phi\left(s^{\prime}\right)=f\left(k s^{\prime}\right)
$$

around the point

$$
a^{\prime}=\frac{a}{k}
$$

obtaining quantities $\alpha_{j}^{\prime}$ as coefficients of $\left(s^{\prime}-a^{\prime}\right)^{i}$, from which, theoretically

$$
\alpha_{j}=\frac{\alpha_{j}^{\prime}}{k^{i}}
$$

Then by comparing the original quantities $\alpha_{j}$, with those obtained from (67), as $j$ increases, one could estimate the cumulative building up of round-off errors.

D. P. Flemming

Canadian Armament

Research and Development Establishment

P. O. Box 1427

Quebec, Que.

The main part of the work connected with the preparation of this paper was done while the author was employed at Minneapolis-Honeywell Regulator Company, Minneapolis, Minnesota.

1. C. K. Tirus, "A general card-program for the evaluation of the inverse Laplace transform," Assn. for Comp. Machinery, Journ., v. 2, 1955, p. 18-27.

2. H. S. CARSLAw \& J. C. JAEGER, Operational Methods in Applied Mathematics, Oxford Univ. Press, 2nd Edition, 1948.

3. WM. FELLER, An Introduction to Probability Theory and its Applications, John Wiley \& Sons, Inc., New York, 1950.

4. R. V. CHURCHILL, Introduction to Complex Variables and Applications, McGraw-Hill Book Co., Inc., New York, 1948.

\section{Numerical Integration over Simplexes and Cones}

1. Introduction. In this paper we develop numerical integration formulas for simplexes and cones in $n$-space for $n \geq 2$. While several papers have been written on numerical integration in higher spaces, most of these have dealt with hyperrectangular regions. For certain exceptions see [3]. Hammer and Wymore [1] have given a first general type theory designed through systematic use of cartesian product regions and affine transformations to extend the possible usefulness of formulas for each region. 